\title{
Time Periodic Solutions of Some Non-linear Evolution Equations
}

By

\author{
Takao KAKITA*
}

\section{§1. Introduction}

Considered in this paper are non-linear evoiution equations of the form

$$
\frac{\partial^{2} u}{\partial t^{2}}+A u+B\left(u, \frac{\partial u}{\partial t}\right)=f(x, t) \quad \text { in } \Omega \times(-\infty, \infty)
$$

together with periodicity conditions

$$
u(x, t)=u(x, t+\tau), \quad u_{t}(x, t)=u_{t}(x, t+\tau)
$$

and Dirichlet boundary conditions

$$
D^{\alpha} u(x, t)=0 \text { on } \partial \Omega \text { for }|\alpha| \leqq m-1 \text {. }
$$

Each $A$ in (1) is a non-linear elliptic operator of order $2 m$ in $\Omega$, a fixed bounded domain in $\boldsymbol{R}^{N}$ (which is similar to the one defined by F.E. Browder ([1])), and

$$
\begin{array}{r}
B\left(u, u_{t}\right)=\beta_{0}^{\prime}\left(|u|^{2}\right) u_{t}-\Delta u_{t}, \text { or more generally } \\
=\sum_{|\alpha| \leq m-1}(-1)^{|\alpha|} D^{\alpha} \beta_{\alpha}^{\prime}\left(\left|D^{\alpha} u\right|^{2}\right) D^{\alpha} u_{t} \\
\left(\beta_{\alpha}^{\prime}\left(s^{2}\right) \geqq \varepsilon_{0}>0 \text { for }|\alpha|=1\right)
\end{array}
$$

where each $\beta_{\alpha}^{\prime}\left(s^{2}\right)$ is a non-negative function on $\mathbb{R}^{1}$, of polynomial growth. Each function $f(x, t)$ on $\Omega \times \mathbb{R}^{1}$ is periodic in $t$ (of period $\tau>0$ ) with values in an appropriate Sobolev space. The purpose of this paper is to prove

Communicated by S. Matsuura, February 8, 1973.

+ Department of Mathematics, School of Science and Engineering, Waseda Iniversity, Tokyo. 
an existence theorem of weak solutions for the equation (1) with conditions (2)-(3), subsequently to [3], where the theorem was proved for $A$, semilinear elliptic operators, and $B\left(u, u_{t}\right)=\left(1+\beta_{0}^{\prime}\left(|u|^{2}\right)\right) u_{t}$. In case $A$ is a quasi-linear elliptic operator of the second order and $B\left(u, u_{t}\right)=\Delta u_{t}=$ $\sum_{i=1}^{N} \frac{\partial^{3} u}{\partial x^{2} \partial t}$, initial-boundary value problems for (1) have been solved by M. Tsutsumi ([7]), J.C. Clements $([2])$, also boundary value problems with periodic conditions for (1) by Clements $([2])$. W. A. Strauss has obtained (in unpublished work) weak solutions periodic in $t$ of the equation

$$
\frac{\partial^{2} u}{\partial t^{2}}-\Delta u+|u|^{p-1} u+|u|^{q-1} \frac{\partial u}{\partial t}=f(x, t)
$$

where $f(x, t)$ is a function periodic in $t, p \geqq q \geqq 1(\mathrm{cf} .[6]){ }^{1)}$

An example of our theorem gives the existence of periodic solutions in $t$ for the equation

$$
\frac{\partial^{2} u}{\partial t^{2}}-\sum_{i=1}^{N} \frac{\partial}{\partial x_{i}}\left(\left|\frac{\partial u}{\partial x_{i}}\right|^{p-2} \frac{\partial u}{\partial x_{i}}\right)-\Delta \frac{\partial u}{\partial t}+|u|^{q-1} \frac{\partial u}{\partial t}=f(x, t)
$$

where $f(x, t)$ is as above and $p / 2 \geqq q \geqq 2$.

\section{§2. Definitions and Main Theorem}

Let $W^{m, p}(\Omega)$ be the Sobolev space

$$
\left\{u(x)\left|D^{\alpha} u(x) \in L^{p}(\Omega),\right| \alpha \mid \leqq m\right\}^{2)}
$$

with norm

$$
\|u\|_{m, p}=\left(\sum_{\alpha \mid \leqq m} \int_{\Omega}\left|D^{\alpha} u(x)\right|^{p} d x\right)^{1 / p}
$$

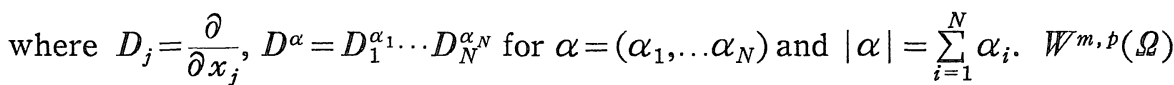
is a separable Banach space for $1<p<\infty . W_{0}^{m, p}(\Omega)$ is the closure of $C_{0}^{\infty}(\Omega)$ (the space of all $C^{\infty}$-functions in $\Omega$ with compace support) in $W^{m, p}(\Omega) . \quad C^{1}(\tau)$ is defined as the set of all periodic functions in $C^{1}\left(\mathbb{R}^{1}\right)$ of

1) This result was informed to the author by Professor Strauss.

2) Throughout the paper we assume all functions considered are real valued. 
period $\tau$. By $\left\langle u^{*}, u\right\rangle$ we denote the value of $u^{*} \in X^{*}$ at $u \in X$ for a Banach space $X$ and its dual $X^{*}$. We denote by $L^{p}(\tau ; X)$ the Banach space of functions $f$ which are in $L^{p}$ over any $I_{\tau}=[t, t+\tau]$ with values in $X$ and

$$
f(t)=f(t+\tau) \quad \text { in } X \text { for all } t \in \mathbb{R}^{1},
$$

provided with the norm $(1 \leqq p<\infty)$

$$
\|f\|_{L^{p}(\tau: X)}=\left\{\int_{I_{\tau}}\|f(t)\|_{X}^{p} d t\right\}^{1 / p}
$$

As for $L^{\infty}(\tau ; X)$, the usual modification is needed. The $L^{p}(\Omega)$ norm is denoted by $\|\cdot\|_{p}$, especially by $\|\cdot\|$ for $p=2$.

Assumption $\mathbb{A}$. Let $A$ be a (non-linear) defferential operator in $\Omega$, given in divergence form

$$
A u(x)=\sum_{|\alpha| \leqq m}(-1)^{|\alpha|} D^{\alpha} A_{\alpha}\left(x, u, \ldots, D^{m} u\right)
$$

where $D^{k} u=\left\{D^{\alpha} u\right\}_{|\alpha|=k}$, and the following conditions are imposed on $A_{\alpha}$ :

i) each $A_{\alpha}(x, \xi)$ is a continuous function of $(x, \xi)$ ( $\xi$ is a real vector corresponding to $\left.\left\{D^{\alpha} u\right\}_{|\alpha| \leqq m}\right)$;

ii) there exists a continuous function $g_{0}(s)$ on $R^{1}$ such that

$$
\left|A_{\alpha}\left(x, u(x), \ldots, D^{m} u(x)\right)\right| \leqq g_{0}\left(\|u\|_{m, p}\right)\left\{\sum_{|\beta| \leqq m}\left|D^{\beta} u(x)\right|^{p-1}+1\right\}
$$

for all $u \in W_{0}^{m, p}(\Omega)(p \geqq 2)$, all $\alpha$ with $|\alpha| \leqq m$ and almost all $x \in \Omega$;

iii) the non-linear Dirichlet form on $\mathbb{W}, 1)$

$$
u(u, v)=\sum_{|\alpha| \leq m} \int_{\Omega} A_{\alpha}\left(x, u(x), \ldots, D^{m} u(x)\right) D^{\alpha} v(x) d x
$$

satisfies, for a continuous function $g_{1}(s) \geqq 0$ on $\mathbb{R}^{2}$,

$$
|a(u, v)| \leqq g_{1}\left(\|u\|_{m, p}\right)\|v\|_{m, p}, \quad u, v \in W
$$

iv) for $u \in W$,

$$
a(u, u) \geqq c_{0}\left(\|u\|_{m, p}\right)+k_{0}\|u\|^{2}
$$

1) We denote $W_{0}^{m \cdot p}(\Omega)$ by $W$ for simplicity. 
where $c_{0}(s)$ is a continuous function on $\mathbb{R}^{1}$ with $\lim _{s \rightarrow \infty} c_{0}(s)=\infty$ and $k_{0}$ is a positive constant;

v) $a(u, u-v)-a(v, u-v) \geqq 0, u, v \in W$;

vi) there exists a functional $r(u)$ on $W$ such that

$$
a\left(\psi(t), \phi^{\prime}(t)\right) \geqq \frac{d}{d t} r(\phi(t))
$$

for any $\psi(t)$, a finite sum of functions $c(t) w$ for $c(t) \in C^{1}(\tau)$ and $w \in W$, and that for $u \in \mathbb{W}$

$$
c_{1}\left(\|u\|_{m, p}\right) \leqq r(u) \leqq k_{1} a(u, u)+k_{2}
$$

where $c_{1}(s)$ is a continuous function on $\boldsymbol{R}^{1}$ with $\lim _{s \rightarrow \infty} c_{1}(s)=\infty$ and $k_{1}, k_{2}$ are some constants.

Assumption B. Let $\beta_{0}(s)$ be a twice differentiable function on $\boldsymbol{R}^{1}$ such that for $|\alpha| \leqq m-1$

$$
\begin{aligned}
0 \leqq & \beta_{\alpha}^{\prime}\left(s^{2}\right) \leqq C|s|^{q-1}, \text { in particular, } \varepsilon_{0} \leqq \beta_{\alpha}^{\prime}\left(s^{2}\right) \quad(|\alpha|=1) \\
& \left|\beta_{\alpha}^{\prime \prime}\left(s^{2}\right)\right| \leqq C|s|^{q-3}
\end{aligned}
$$

where $2 \leqq q \leqq p / 2, C, \varepsilon_{0}$ are constants $>0$.

Now our theorem is stated as follows.

Theorem. Given $f(t) \in L^{2}(\tau ; W)$ (not zero), there exists a solution $u \in L^{\infty}(\tau ; W)$ of the equation (1):

$$
u_{t t}+A u+B\left(u, u_{\imath}\right)=f
$$

such that $u_{t} \in L^{2}\left(\tau ; L^{2}(\Omega)\right), u_{t t} \in L^{p^{\prime}}\left(\tau ; \mathbb{W}^{*}\right)$ and that

$$
B\left(u, u_{t}\right)=\sum_{|\alpha| \leqq m-1}(-1)^{|\alpha|} D^{\alpha} \beta_{\alpha}^{\prime}\left(\left|D^{\alpha} u\right|^{2}\right) D^{\alpha} u_{t}
$$

where $1 / p+1 / p^{\prime}=1, A$ and $B$ satisfy Assumption A and Assumption B, respectively.

\section{§3. Proof of the Theorem}

We shall prove the theorem by means of Faedo-Galerkin's method 
combined with the fixed point theorem and the compactness method. Since $W$ is separable, there exists a countable basis $\left\{w_{n}\right\}$ in $W$ which is orthonormal in $L^{2}(\Omega)$. Let $W_{n}$ be the subspace of $W$ spanned by $w_{1}, \ldots, w_{n}$.

Consider the ordinary differential system in $\mathbb{W}_{n}$

$$
\begin{aligned}
& \left(u_{t l}^{n}(t), w_{j}\right)+a\left(u^{n}(t), w_{j}\right)+b\left(u^{n}(t), u_{t}^{n}(t) ; w_{j}\right) \\
& =\left(f(t), w_{j}\right) \quad(j=1,2, \ldots, n)
\end{aligned}
$$

with periodic conditions

$$
u^{n}(t)=u^{n}(t+\tau), \quad u_{t}^{n}(t)=u_{t}^{n}(t+\tau)
$$

where

$$
\begin{aligned}
b\left(u^{n}(t), u_{t}^{n}(t) ; w_{j}\right) & =\left(B\left(u^{n}(t), u_{t}^{n}(t)\right), w_{j}\right) \\
& =\left(\beta_{0}^{\prime}\left(\left|u^{n}(t)\right|^{2}\right) u_{t}^{n}(t), w_{j}\right)+\left(\left(u_{t}^{n}, w_{j}\right)\right) .
\end{aligned}
$$

The solutions will be of the form

$$
u^{n}(t)=\sum_{k=1}^{n} c_{n, k}(t) w_{k}, \quad c_{n, k}(t) \in C^{1}(\tau)
$$

if they exist. Now the substitution of the $u^{n}(t)$ into (10), (11) gives the second order differential system of $\left.\boldsymbol{C}_{n}(t)=\left(c_{n 1}(t), \ldots, c_{n n}(t)\right)^{*}, 1\right)$

$$
\mathbb{C}_{n}^{\prime \prime}(t)+\mathbb{F}\left(\boldsymbol{C}_{n}(t)\right)+\mathbb{H}\left(\mathbb{C}_{n}(t), \quad \mathbb{C}_{n}^{\prime}(t)\right)=\mathbb{H}_{0}(t)
$$

and the periodic conditions

$$
\boldsymbol{C}_{n}(t)=\boldsymbol{C}_{n}(t+\tau), \quad \boldsymbol{C}_{n}^{\prime}(t)=\boldsymbol{C}_{n}^{\prime}(t+\tau),
$$

where

$$
\begin{aligned}
& \boldsymbol{F}\left(\boldsymbol{C}_{n}(t)\right)=\left(\boldsymbol{F}_{1}\left(\boldsymbol{C}_{n}(t)\right), \ldots F_{n}\left(\mathbb{C}_{n}(t)\right)\right)^{*}, \\
& F_{j}\left(\boldsymbol{C}_{n}(t)\right)=a\left(u^{n}(t), w_{j}\right) ; \\
& \boldsymbol{H}\left(\boldsymbol{C}_{n}(t), \boldsymbol{C}_{n}^{\prime}(t)\right)=\left(H_{1}\left(\mathbb{C}_{n}(t)\right), \ldots H_{n}\left(\mathbb{C}_{n}(t)\right)\right)^{*},
\end{aligned}
$$

1) * denotes the transpose operation of $n$-vector. 


$$
\begin{array}{r}
H_{j}\left(\mathbb{C}_{n}(t), \mathbb{C}_{n}^{\prime}(t)\right)=b\left(u^{n}(t), u_{t}^{n}(t) ; w_{j}\right) ; \\
\mathbb{H}_{0}(t)=\left(f_{1}(t), \ldots, f_{n}(t)\right)^{*}, f_{j}(t)=\left(f(t), w_{j}\right) \\
\quad(j=1,2, \ldots, n)
\end{array}
$$

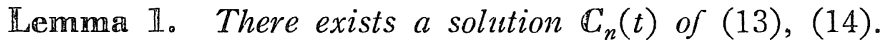

Proof. Consider a system of $\lambda$ dependence $(0 \leqq \lambda \leqq 1)$,

$$
\begin{aligned}
\mathbb{C}_{n}^{\prime \prime}(t)+\delta \mathbb{C}_{n}^{\prime}(t)+k \mathbb{C}_{n}(t) \\
=\lambda\left\{-\mathbb{F}\left(\mathbb{C}_{n}(t)\right)+k \mathbb{C}_{n}(t)-\mathbb{H}\left(\mathbb{C}_{n}(t), \mathbb{C}_{n}^{\prime}(t)\right)+\delta \mathbb{C}_{n}^{\prime}(t)\right\} \\
\quad+\mathbb{H}_{0}(t)
\end{aligned}
$$

together with (14). Here $\delta$ and $k$ are any fixed constants such that $0<\delta<\delta_{0}, 0<k<k_{0}$ where $\delta_{0}$ is a constant satisfying $\delta_{0}\|u\| \leqq\|u\|_{1,2}$ for any $u \in H^{1}(\Omega), k_{0}$ a constant in Assumption A-(iv). Let $G_{n}(t, s)$ be a unique Green's function of (15) for $\lambda=0, \mathbb{H}_{0}=0$ with conditions (14), and define the operator $T_{n}(\lambda)$ from a Banach space $X_{n}$ into itself:

$$
\begin{aligned}
T_{n}(\lambda) \mathbb{C}_{n}= & \int_{I_{\mathrm{r}}}\left[\lambda\left\{-\mathbb{F}\left(\mathbb{C}_{n}(s)\right)+k \mathbb{C}_{n}(s)-\mathbb{H}\left(\mathbb{C}_{n}(s), \mathbb{C}_{n}^{\prime}(s)\right)+\delta \mathbb{C}_{n}^{\prime}(s)\right\}\right. \\
& \left.+\mathbb{H}_{0}(s)\right] G_{n}(t, s) d s
\end{aligned}
$$

where

$$
X_{n}=\mathbb{C}^{1}(\tau) \times \cdots \times \mathbb{C}^{1}(\tau) \quad(n \text {-copies })
$$

with norm

$\left\|\mathbb{C}_{n}\right\|_{X_{n}}=\sup _{I_{\mathrm{r}}}\left\{\left|\mathbb{C}_{n}(t)\right|+\left|\mathbb{C}_{n}^{\prime}(t)\right|\right\}(|\cdot|:$ the length of $n$-vector $)$ for $\mathbb{C}_{n} \in X_{n}$.

To prove the lemma it suffices to show that the operator $T_{n}(1)$ has a fixed point in $X_{n}$. So we apply Leray-Schauder's theorem to the family of operators $T_{n}(\lambda)(0 \leqq \lambda \leqq 1)$ on the space $X_{n}$. We observe that

$$
\left\|F\left(\mathbb{C}_{n}^{(\nu)}\right)-F\left(\mathbb{C}_{n}\right)\right\|_{\infty} \rightarrow 0
$$

and

$$
\left\|H\left(\mathbb{C}_{n}^{(\nu)}, \mathbb{C}_{n}^{(\nu)^{\prime}}\right)-H\left(\mathbb{C}_{n}, \mathbb{C}_{n}^{\prime}\right)\right\| \rightarrow 0
$$


when $\mathbb{C}_{n}^{(\nu)} \rightarrow \mathbb{C}_{n}$ in $X_{n}$, as $\nu \rightarrow \infty$. In fact, (17) is a direct consequence of Assumption $\mathrm{A}$ on $A_{\alpha}$ by measure theoretical arguments. To show (18) we put

$$
\begin{aligned}
& u^{(\nu)}(x, t)=\sum_{k=1}^{n} c_{k}^{(\nu)}(t) w_{k}(x) \\
& u(x, t)=\sum_{k=1}^{n} c_{k}(t) w_{k}(x)
\end{aligned}
$$

dropping the suffix $n$ for brevity in notation. Assumption B implies

$$
\begin{aligned}
& \left|\beta_{0}^{\prime}\left(\left|u^{(\nu)}(x, t)\right|^{2}\right)-\beta_{0}^{\prime}\left(|u(x, t)|^{2}\right)\right| \\
& \quad \leqq C\left|u^{(\nu)}(x, t)-u(x, t)\right|\left(\left|u^{(\nu)}(x, t)\right|+|u(x, t)|\right)^{q-2}
\end{aligned}
$$

for all $t$ and almost all $x \in \Omega$. Since $\left\|\mathbb{C}^{(\nu)}\right\|_{\infty},\left\|\mathbb{C}^{(\nu)^{\prime}}\right\|_{\infty}$ are bounded on $\nu$, we obtain by Hölder's inequality

$$
\begin{aligned}
& \left|\left(u_{t}^{(\nu)}(x, t)\left(\beta_{0}^{\prime}\left(\left|u^{(\nu)}(x, t)\right|^{2}\right)-\beta_{0}^{\prime}\left(|u(x, t)|^{2}\right)\right), w_{j}\right)\right| \\
& \leqq C\left\|u_{t}^{(\nu)}(t)\right\| \cdot\left\|u^{(\nu)}(t)-u(t)\right\|_{2 q}\left(\left\|u^{(\nu)}(t)\right\|_{2 q}^{q-2}+\|u(t)\|_{2 q}^{q-2}\right)\left\|w_{j}\right\|_{2 q} \\
& \leqq K\left\|\mathbb{C}^{(\nu)}-\mathbb{C}\right\|_{X_{n}}
\end{aligned}
$$

for some constant $K$. Similarly we have

$$
\left|\left(\left(u_{t}(x, t)-u_{t}^{(\nu)}(x, t)\right) \beta^{\prime}\left(|u(x, t)|^{2}\right), w_{j}\right)\right| \leqq K|| \mathbb{C}^{(\nu)}-\mathbb{C} \|_{X_{n}}
$$

Hence $\left(u_{t}^{(\nu)}(t) \beta_{0}^{\prime}\left(\left|u^{(\nu)}(t)\right|^{2}\right), w_{j}\right) \rightarrow\left(u_{t}(t) \beta_{0}^{\prime}\left(|u(t)|^{2}\right), w_{j}\right)$ uniformly on $t$ as $\nu \rightarrow \infty$, which implies (18). Thus the continuity of $T_{n}(\lambda)$ on $X_{n}$ follows immediately from (16), (17) and (18).

Next, let $S=\left\{\mathbb{C} \in X_{n}|| \mid \mathbb{C}_{n} \| \leqq 1\right\}$. Then the properties of $G_{n}(t, s)$ imply that for each $\lambda, T_{n}(\lambda) S$ is bounded in $X_{n}$ and is a set of equi-continuous functions, and that

$$
\|\left.\left(T_{n}\left(\lambda_{2}\right)-T_{n}\left(\lambda_{1}\right)\right) S\right|_{X_{n}} \leqq K\left|\lambda_{2}-\lambda_{1}\right|
$$

for a suitable constant $K$. Therefore each $T_{n}(\lambda)$ is a compact operator from $X_{n}$ into $X_{n}$ and the family $\left\{T_{n}(\lambda) \mid 0 \leqq \lambda \leqq 1\right\}$ is homotopic. We note that the topological degree of $T_{n}(0)$ is +1 since the system (15) for $\lambda=0$ has a unique solution in $X_{n}$. In order to see that the topological degree 
of $T_{n}(1)$ is +1 (positive) it only remains to show that for each $\lambda$

$$
\mathbb{C}(t)=T_{n}(\lambda) \boldsymbol{C}(t) \Rightarrow\|\mathbb{C}\|_{X_{n}} \leqq L
$$

where $L$ is a constant independent of $\lambda$. The proof of (19) is a variant of that of the following lemma, and is omitted.

Q.E.D.

Lemma 2. The solutions $u^{n}$ of (10), (11) have the following estimates:

$$
\begin{gathered}
\sum_{|\alpha| \leqq 1} \int_{I_{\mathrm{r}}}\left\|D^{\alpha} u_{t}^{n}(t)\right\|^{2} d t \leqq K_{1}, \\
\left\|u_{t}^{n}(t)\right\|,\left\|u^{n}(t)\right\|_{m, p} \leqq K_{2}
\end{gathered}
$$

where $K_{1}, K_{2}$ are constants independent of $n$ and $t$.

Proof. Since both sides of (10) are linear on $w_{j}$, we have, replacing $w_{j}$ by $u_{t}^{n}$,

$$
\begin{aligned}
& \left(u_{t t}^{n}(t), u_{t}^{n}(t)\right)+a\left(u^{n}(t), u_{t}^{n}(t)\right)+b\left(u^{n}(t), u_{t}^{n}(t)\right) \\
& =\left(f(t), u_{t}^{n}(t)\right) .
\end{aligned}
$$

Integrating both sides over $I_{\tau}$ with respect to $t$ and using Assumptions A-(vi), B we obtain

$$
\sum_{|\alpha| \leqq 1} \int_{I_{\mathrm{r}}}\left\|D^{\alpha} u_{t}^{n}(t)\right\|^{2} d t \leqq\left(\int_{I_{\mathrm{r}}}\|f(t)\|^{2} d t\right)^{1 / 2}\left(\int_{I_{\mathrm{r}}}\left\|u_{t}^{n}(t)\right\|^{2} d t\right)^{1 / 2}
$$

from which the estimates (20) follows immediately.

Replacing $w_{j}$ by $u^{n}$ in (10) gives

$$
\begin{aligned}
& \left(u_{t t}^{n}(t), u^{n}(t)\right)+a\left(u^{n}(t), u^{n}(t)\right) \\
& +b\left(u^{n}(t), u_{t}^{n}(t) ; u^{n}(t)\right)=\left(f(t), u^{n}(t)\right) .
\end{aligned}
$$

We remark that

$$
\int_{I_{\mathbf{r}}} b\left(u^{n}(t), u_{t}^{n}(t) ; u^{n}(t)\right) d t=0
$$

because of the periodicity of $u^{n}(t)$. Then, integrating (22) over $I_{\tau}$ with respect to $t$ and using Assumption A-(iv) we have 


$$
\begin{aligned}
& k_{0} \int_{I_{\mathrm{r}}}\left\|u^{n}(t)\right\|^{2} d t+\int_{I_{\mathrm{r}}} c_{0}\left(\left\|u^{n}(t)\right\|_{m, p}\right) d t \\
& \leqq \int_{I_{\mathrm{r}}}\left\|u_{t}^{n}(t)\right\|^{2} d t+\left(\int_{I_{\mathrm{r}}}\|f(t)\|^{2} d t\right)^{1 / 2}\left(\int_{I_{\mathrm{r}}}\left\|u^{n}(t)\right\|^{2} d t\right)^{1 / 2} .
\end{aligned}
$$

This yields that

$$
\int_{I_{\mathrm{r}}}\left\|u^{n}(t)\right\|^{2} d t, \quad \int_{I_{\mathrm{r}}} a\left(u^{n}(t), u^{n}(t)\right) d t
$$

have a bound independent of $n$, because $\int_{I_{\mathrm{r}}} c_{0}\left(\left\|u^{n}(t)\right\|_{m, p}\right) d t$ is bounded from below on $n$.

Finally substituting $u_{t}^{n}$ for $w_{j}$ in (10) and integrating both sides from $s$ to $t(s<t)$, we obtain by Assumption A-(vi),

$$
\begin{aligned}
& \frac{1}{2}\left\|u_{t}^{n}(t)\right\|^{2}+c_{1}\left(\left\|u^{n}(t)\right\|_{m, p}\right) \\
& \leqq K+\frac{1}{2}\left\|u_{t}^{n}(s)\right\|^{2}+k_{1} a\left(u^{n}(s), u^{n}(s)\right)+k_{2}
\end{aligned}
$$

where $K$ is a constant dependent of $f$ and $K_{1}$ in (20). Further, integrating both sides of (25) with respect to $s$ from $t-\tau$ to $t$ and noting that the right hand side is bounded by virtue of (24), we know that

$$
\left\|u_{t}^{n}(t)\right\|_{1}, c_{1}\left(\left\|u^{n}(t)\right\|_{m, p}\right) \leqq K \quad \text { (independent of } n \text { and } t \text { ). }
$$

Since $\lim _{s \rightarrow \infty} c_{1}(s)=\infty$, this proves the lemma.

Q.E.D.

Now we may infer that

$\left\{u^{n}\right\}$ is bounded in $L^{\infty}(\tau ; W)$,

$\left\{u_{t}^{n}\right\}$ is bounded in $L^{2}\left(\tau ; H^{1}(\Omega)\right) \cap L^{\infty}\left(\tau ; L^{2}(\Omega)\right)$.

Then we may extract a subsequence $\left\{u^{\nu}\right\}$ such that

$$
\begin{aligned}
& \left.u^{\nu} \rightarrow u \text { (an element of } L^{\infty}(\tau ; W)\right) \text { weakly star in } L^{\infty}(\tau ; W), \\
& u_{t}^{\nu} \rightarrow u_{t} \text { strongly in } L^{2}\left(\tau ; L^{2}(\Omega)\right) ;
\end{aligned}
$$

in addition, 
$u^{\nu} \rightarrow u$ strongly in $L^{p}\left(\Omega \times \mathbb{I}_{\tau}\right)$

where we have used that the injection mappings

$$
\begin{aligned}
& i: W^{k, p}(\Omega) \rightarrow W^{k-1, p}(\Omega) \\
& j: H^{1}(\Omega) \rightarrow L^{2}(\Omega)
\end{aligned}
$$

are compact.

Making use of these results, we shall prove:

Lemma 3. For any $v \in L^{2}(\tau ; W)$

$$
\int_{I_{\tau}} b\left(u^{\nu}, u_{t}^{\nu} ; v\right) d t \rightarrow \int_{I_{\tau}} b\left(u, u_{t} ; v\right) d t
$$

Proof. By definition, we have

$$
\begin{aligned}
& b\left(u^{\nu}, u_{t}^{\nu} ; v\right)-b\left(u, u_{t} ; v\right) \\
& =\left(u_{t}^{\nu}-u_{t}, \beta_{0}^{\prime}\left(|u|^{2}\right) v\right)+\left(u_{t}^{\nu},\left(\beta_{0}^{\prime}\left(\left|u^{\nu}\right|^{2}\right)-\beta_{0}^{\prime}\left(|u|^{2}\right)\right) v\right)
\end{aligned}
$$

Assumption B implies

$$
\left|\beta_{0}^{\prime}\left(|u(t)|^{2}\right) v(t)\right| \leqq C|u(t)|^{q-1}|v(t)| .
$$

Therefore we obtain

$$
\int_{\Omega}|u(t)|^{2(q-1)}|v(t)|^{2} d x \leqq\|u(t)\|_{2 q}^{2(p-1)}\|v(t)\|_{2 q}^{2},
$$

which means

$$
\beta_{0}^{\prime}\left(|u(t)|^{2}\right) v(t) \in L^{2}\left(\tau ; L^{2}(\Omega)\right)
$$

As $u_{t}^{\nu} \rightarrow u_{t}$ strongly in $L^{2}\left(\tau ; L^{2}(\Omega)\right)$, we know that

$$
\int_{I_{\mathrm{r}}}\left(u_{t}^{\nu}-u_{t}, \beta_{0}^{\prime}\left(|u|^{2}\right) v\right) d t \rightarrow 0
$$

For the second term of the right hand side of (26), since

$$
\left|\beta_{0}^{\prime}\left(\left|u^{\nu}\right|^{2}\right)-\beta_{0}^{\prime}\left(|u|^{2}\right)\right| \leqq\left|u^{\nu}-u\right|\left(\left|u^{\nu}\right|^{q-2}+|u|^{q-2}\right)
$$

we obtain 


$$
\begin{aligned}
& \left|\left(u_{t}^{\nu},\left(\beta_{0}^{\prime}\left(\left|u^{\nu}\right|^{2}\right)-\beta_{0}^{\prime}\left(|u|^{2}\right)\right) v\right)\right| \\
& \quad \leqq C\left\|u_{t}^{\nu}(t)\right\| \cdot\left\|u^{\nu}(t)-u(t)\right\|_{2 q}\left(\left\|_{1}^{\nu} u^{\nu}(t)\right\|_{2 q}^{q-2}+\|u(t)\|_{2 q}^{q-2}\right)\|v(t)\|_{2 q},
\end{aligned}
$$

taking boundedness of $\left\|u^{\nu}(t)\right\|_{2 q}^{q-2},\left\|u_{t}^{\nu}(t)\right\|$ into consideration,

$$
\leqq K\left\|u^{\nu}(t)-u(t)\right\|_{2 q}\|v(t)\|_{2 q}
$$

where $K$ is a constant independent of $n$ and $t$. Therefore we have

$$
\begin{aligned}
& \left|\int_{I_{\tau}}\left(u_{t}^{\nu},\left(\beta_{0}^{\prime}\left(\left|u^{\nu}\right|^{2}\right)-\beta_{0}^{\prime}\left(|u|^{2}\right)\right) v\right) d t\right| \\
& \leqq K\left(\int_{I_{\tau}}\left\|u^{\nu}(t)-u(t)\right\|_{2 q}^{2} d t\right)^{1 / 2}\left(\int_{I_{\tau}}\|v(t)\|_{2 q}^{2} d t\right)^{1 / 2} \\
& \leqq K_{1}\left\|u^{\nu}-u\right\|_{L^{2 q\left(\Omega \times I_{\tau}\right)}} \cdot\|v\|_{L^{2}\left(\tau ; L^{2 q}(\Omega)\right)},
\end{aligned}
$$

$K_{1}$ being another constant independent of $n$. Since $u^{\nu} \rightarrow u$ strongly in $L^{p}\left(\Omega \times I_{\tau}\right)$, the last member in the above inequalities tends to zero. Thus we proved the lemma.

Q.E.D.

Finally, to establish the remaining part of our theorem, we need the following assertion.

Lemma 4. There exists a subsequence $\{\mu\}$ of $\{\nu\}$ such that

$$
\int_{I_{\mathrm{r}}} a\left(u^{\mu}(t), v(t)\right) d t \rightarrow \int_{I_{\mathrm{r}}} a(u(t), v(t)) d t \quad(\mu \rightarrow \infty)
$$

for any $v \in L^{2}(\tau ; W)$.

Proof. Consider a linear form on $L^{2}(\tau ; W)$ :

$$
v \rightarrow \int_{I_{\mathrm{r}}} a(u(t), v(t)) d t
$$

Since $u \in L^{\infty}(\tau ; W)$, Assumption A-(iii) implies that

$$
\begin{aligned}
\left|\int_{I_{\tau}} a(u(t), v(t)) d t\right| & \leqq \int_{I_{\tau}} g_{1}\left(\|u(t)\|_{m, p}\right)\|v(t)\|_{m, p} d t \\
& \leqq K\|v\|_{L^{2}(\tau ; W)} .
\end{aligned}
$$

Hence the linear form is continuous on $L^{2}(\tau ; W)$, so that there is an 
element $\mathscr{A} u \in L^{2}\left(\tau ; W^{*}\right)$ such that

$$
\int_{I_{\mathrm{r}}} a(u(t), v(t)) d t=\langle\langle\mathscr{A} u, v\rangle .
$$

Here $《, 》$ denotes the pairing between $L^{2}(\tau ; W)$ and $L^{2}\left(\tau ; W^{*}\right)$. The operator $\mathscr{A}$ sending $L^{2}(\tau ; W)$ into $L^{2}\left(\tau ; W^{*}\right)$ satisfies

$$
\|\mathscr{A} u\|_{L^{2}(\tau ; W)}=\sup _{v}\left|\int_{I_{\mathrm{r}}} a\left(u^{\nu}(t), v(t)\right) d t\right| \leqq K
$$

where $v$ in the second member runs through the set $\left\{\|v\|_{L^{2}(\tau ; W)} \leqq 1\right\}$. Thus there exist a subsequence $\{\mu\} \subset\{\nu\}$ and an element $\xi \in L^{2}\left(\tau ; W^{*}\right)$ such that for $v \in L^{2}(\tau ; W)$

$$
\int_{I_{\mathrm{r}}} a\left(u^{\mu}(t), v(t)\right) d t \rightarrow\langle\xi, v\rangle
$$

where $\xi$ is an element of $L^{2}\left(\tau ; W^{*}\right)$. We assert $\mathscr{A} u=\xi$. Take any $\varphi$, a finite sum of $c_{k}(t) w_{k}(x)$ where $c_{k} \in C^{1}(\tau), w_{k} \in W$. Then for large $n$ hold the equalities:

$$
\begin{aligned}
& -\int_{I_{\tau}}\left(u_{t}^{\mu}(t), \varphi_{t}(t)\right) d t+\int_{I_{\mathrm{r}}} a\left(u^{\mu}(t), \varphi(t)\right) d t \\
& +\int_{I_{\tau}} b\left(u^{\mu}(t), u_{t}^{\mu}(t) ; \varphi(t)\right) d t=\int_{I_{\mathrm{r}}}(f(t), \varphi(t)) d t .
\end{aligned}
$$

Letting $\mu \rightarrow \infty$, we have

$$
\begin{aligned}
-\int_{I_{\mathrm{r}}}\left(u_{t}(t), \varphi_{t}(t)\right) d t+\langle\xi, \varphi\rangle & +\int_{I_{\mathrm{r}}} b\left(u(t), u_{t}(t) ; \varphi(t)\right) d t \\
& =\int_{I_{\mathrm{r}}}(f(t), \varphi(t)) d t
\end{aligned}
$$

Let

$$
V(\tau ; W)=\left\{\nu \in L^{2}(\tau ; W) \mid v_{t} \in L^{2}\left(\tau ; L^{2}(\Omega)\right)\right\}
$$

with norm

$$
\|v\|_{V(\tau ; W)}=\|v\|_{L^{2}(\tau ; W)}+\left\|v_{t}\right\|_{L^{2}\left(\tau ; L^{2}(\Omega)\right)} .
$$


Since the set of the $\varphi$ defined above is dense in $V(\tau ; W),(27)$ is valid for any $\varphi \in V(\tau ; W)$, in particular, for $\varphi=u$. Hence,

$$
\begin{aligned}
-\int_{I_{\mathrm{r}}}\left\|u_{t}(t)\right\|^{2} d t+\langle\xi, u\rangle & +\int_{I_{\mathrm{r}}} b\left(u(t), u_{t}(t) ; u(t)\right) d t \\
& =\int_{I_{\tau}}(f(t), u(t)) d t
\end{aligned}
$$

However, we observe that

$$
\begin{aligned}
\int_{I_{\tau}}\left(u_{t} \beta_{0}^{\prime}\left(|u|^{2}\right), u\right) d t & =\lim \int_{I_{\tau}}\left(u_{t}^{\mu} \beta_{0}^{\prime}\left(\left|u^{\mu}\right|^{2}\right), u^{\mu}\right) d t \\
& =0,
\end{aligned}
$$

from which follows

$$
-\int_{I_{\tau}}\left\|u_{t}\right\|^{2} d t+\langle\xi, u\rangle=\int_{I_{\tau}}(f, u) d t
$$

Since

$$
-\int_{I_{\mathfrak{r}}}\left\|u_{t}^{\mu}\right\|^{2} d t+\int_{I_{\mathrm{r}}} a\left(u^{\mu}, u^{\mu}\right) d t=\int_{I_{\mathrm{r}}}\left(f, u^{\mu}\right) d t
$$

taking the limit inferior of both sides, and recalling that $u_{t}^{\mu} \rightarrow u_{t}$ strongly in $L^{2}\left(\tau ; L^{2}(\Omega)\right)$ we obtain that

$$
-\int_{I_{\mathrm{r}}}\left\|u_{t}\right\|^{2} d t+\underline{\lim }\left\langle\left\langle\mathscr{A} u^{\mu}, u^{\mu}\right\rangle \leqq \int_{I_{\mathrm{r}}}(f, u) d t .\right.
$$

Comparing (29) with (28) yields

$$
\left\langle\xi \xi u 》 \geqq \underline{\lim }\left\langle\left\langle\mathscr{A} u^{\mu}, u^{\mu}\right\rangle\right.\right.
$$

from which we can conclude in the same way as in [5] that $\xi=\mathscr{A} u$.

Q.E.D.

Now we observe in the proof of Lemma 3 that $B\left(u, u_{t}\right) \in L^{2}\left(\tau ; W^{*}\right)$, so that for any smooth function $\varphi(x, t)$ periodic in $t$, we have in the distributional sense that 


$$
\begin{aligned}
& \int_{I_{\mathrm{r}}}\left(u_{t t}, \varphi\right) d t+\int_{I_{\mathrm{r}}}(A u, \varphi) d t+\int_{I_{\mathrm{r}}}\left(B\left(u, u_{t}\right), \varphi\right) d t \\
& =\int_{I_{\tau}}(f, \varphi) d t \\
& u_{t t}=-A u-B\left(u, u_{t}\right)+f \in L^{2}\left(\tau ; \mathbb{W}^{*}\right)
\end{aligned}
$$

which completes the proof of our theorem, for $B\left(u, u_{t}\right)=\beta_{0}^{\prime}\left(|u|^{2}\right) u_{t}-\Delta u_{t}$. When $B\left(u, u_{t}\right)=\sum_{|\alpha| \leq m-1}(-1)^{|\alpha|} D^{\alpha} \beta_{\alpha}^{\prime}\left(\left|D^{\alpha} u\right|^{2}\right) D^{\alpha} u_{t}$, we need some modifications. Consider the system (15) for $\varepsilon_{0} \delta$ instead of $\delta$ and for

$$
b\left(u^{n}, u_{t}^{n} ; w_{j}\right)=\sum_{|\alpha| \leqq m-1}\left(\beta_{\alpha}^{\prime}\left(\left|D^{\alpha} u^{n}\right|^{2}\right) D^{\alpha} u^{n}, D^{\alpha} w_{j}\right)
$$

Then we can obtain the estimates

$$
\sum_{|\alpha| \leq m-1} \int_{I_{\mathrm{r}}} \|\left. D^{\alpha} u_{t}^{n}\right|^{2} d t \leqq K_{1}
$$

as in the proof of Lemma 2. Also we know by (20)

$$
\sum_{|\alpha| \leqq m} \int_{I_{\tau}}\left\|D^{\alpha} u^{n}\right\|_{p}^{p} d t \leqq K_{2}
$$

Therefore we may choose a further subsequence $\{\sigma\}$ of $\{\mu\}$ such that when $\sigma \rightarrow \infty$,

$$
D^{\alpha} u_{t}^{\sigma} \rightarrow D^{\alpha} u_{t} \quad \text { weakly in } L^{2}\left(\Omega \times I_{\tau}\right)
$$

and

$$
D^{\alpha} u^{\sigma} \rightarrow D^{\alpha} u \quad \text { strongly in } L^{p}\left(\Omega \times \mathbb{I}_{\tau}\right),
$$

both for $|\alpha| \leqq m-1$. Since, for $v \in L^{p}(\tau ; W)$,

$$
\begin{aligned}
& b\left(u^{\sigma}, u_{t}^{\sigma} ; v\right)-b\left(u, u_{t} ; v\right) \\
& =\sum_{|\alpha| \leqq m-1}\left(D^{\alpha} u_{t}^{\sigma}-D^{\alpha} u_{t}, \beta_{\alpha}^{\prime}\left(\left|D^{\alpha} u\right|^{2}\right) D^{\alpha} v\right) \\
& \quad+\sum_{|\alpha| \leqq m-1}\left(D^{\alpha} u_{t}^{\sigma}\left(\beta_{\alpha}^{\prime}\left(\left|D^{\alpha} u^{\sigma}\right|^{2}\right)-\beta_{\alpha}^{\prime}\left(\left|D^{\alpha} u\right|^{2}\right)\right), D^{\alpha} v\right),
\end{aligned}
$$

to prove Lemma 3 for $v \in L^{p}(\tau ; W)$ it is enough to show that for each $\alpha$ 


$$
\begin{aligned}
& \int_{I_{\tau}}\left(D^{\alpha} u_{t}^{\sigma}-D^{\alpha} u_{t}, \beta_{\alpha}^{\prime}\left(\left|D^{\alpha} u\right|^{2}\right) D^{\alpha} v\right) d t \rightarrow 0 \\
& \int_{I_{\tau}}\left(D^{\alpha} u_{t}^{\sigma}\left(\beta_{\alpha}^{\prime}\left(\left|D^{\alpha} u^{\sigma}\right|^{2}\right)-\beta_{\alpha}^{\prime}\left(\left|D^{\alpha} u\right|^{2}\right)\right), D^{\alpha} v\right) d t \rightarrow 0
\end{aligned}
$$

as $\sigma \rightarrow \infty$.

The first assertion is obvious because of (32) and $\beta_{\alpha}^{\prime}\left(\left|D^{\alpha} u\right|^{2}\right) D^{\alpha} v \in L^{2}\left(\Omega \times I_{\tau}\right)$. For the second one, we can show as in the proof of Lemma 3 that

$$
\begin{aligned}
& \mid \int_{I_{\mathfrak{r}}}\left(D^{\alpha} u_{t}^{\sigma}\left(\beta_{\alpha}^{\prime}\left(\left|D^{\alpha} u\right|^{2}\right)-\beta_{\alpha}^{\prime}\left(\left|D^{\alpha} u\right|^{2}\right), D^{\alpha} v\right) d t \mid\right. \\
& \leqq C_{\|}^{\prime} \mid D^{\alpha} u_{t}^{\sigma}\left\|_{L^{2}(\Omega \times I)}\right\| D^{\alpha} u^{\sigma}-D^{\alpha} u \|_{L^{2 q\left(\Omega \times I_{\mathrm{r}}\right)}} \\
& \quad \times\left\{\left\|D^{a} u^{\sigma}\right\|_{L^{2 q\left(\Omega \times I_{\mathrm{r}}\right)}}^{q-\bar{q}^{2}}+\left\|D^{\alpha} u\right\|_{L^{2} q\left(\Omega \times I_{\tau}\right)}^{q}\right\}\left\|D^{\alpha} v\right\|_{L^{2 q\left(\Omega \times I_{\tau}\right)}}^{2} .
\end{aligned}
$$

from which (34) follows by virtue of (31), (33). Since Lemma 4 holds for $v \in L^{p}(\tau ; W)$ we have completed the proof of the theorem.

Example 1. Define $A_{\alpha}, a(u, v)$ by

$$
A_{\alpha}\left(x, u, \ldots, D^{m} u\right)=\left|D^{\alpha} u\right|^{p-2} D^{\alpha} u
$$

and

$$
a(u, v)=\int_{\Omega} \sum_{|\alpha| \leqq m}\left|D^{\alpha} u\right|^{p-2} D^{\alpha} u D^{\alpha} v d x
$$

respectively. It can be easily seen that $A_{\alpha}$ and $a(u, v)$, then, satisfy Assumption A. Hence an evolution equation

$$
\frac{\partial^{2} u}{\partial t^{2}}+\sum_{|a| \leqq m}(-1)^{|\alpha|} D^{\alpha}\left(\left|D^{\alpha} u\right|^{p-2} D^{\alpha} u\right)-\Delta \frac{\partial u}{\partial t}+|u|^{q-1} \frac{\partial u}{\partial t}=f(x, t)
$$

has a solution $u(x, t)$ in $L^{\infty}\left(\tau ; \mathbb{W}_{0}^{m, p}(\Omega)\right)$ provided $2 \leqq q \leqq p / 2$ and $f(x, t) \in L^{2}\left(\tau ; L^{2}(\Omega)\right)$.

Example 2. Let $A$ be the operator defined in Example 1. Then an evolution equation

$$
\frac{\partial^{2} u}{\partial t^{2}}+A u+\sum_{|\alpha| \leqq m-1}(-1)^{|\alpha|}\left|D^{\alpha} u\right|^{q-1} D^{\alpha} \frac{\partial u}{\partial t}-\Delta \frac{\partial u}{\partial t}=f(x, t)
$$


has a solution $u(x, t)$ in $L^{\infty}\left(\tau ; W_{0}^{m, p}(\Omega)\right)$ provided $2 \leqq q \leqq p / 2$ and $f(x, t)$ $\in L^{2}\left(\tau ; L^{2}(\Omega)\right)$.

\section{References}

[1] Browder, F.E., Non-linear elliptic boundary value problems, Bull. Amer. Math. Soc. 69 (1963), 862-874.

[2] Clements, J.C., Existence theorems for a quasilinear evolution equation, to appear.

[3] Kakita, T., On the existence of time-periodic solutions of some non-linear evolution equations, to appear.

[4] Leray J. and Schauder, J., Topologie et equations fonctionnelles, Ann. l'Ecole Norm. Sup., 51 (1934), 45-78.

[5] Lions J.L. and Strauss, W.A., Some non-linear evolution equations, Bull. Soc. Math. France 93 (1965), 43-96.

[6] Strauss. W.A., The energy method in non-linear partial differential equations, Lecture notes, Brasil (1969),

[7] Tsutsumi, M., Some nonlinear evolution equations of second order, Proc. Japan Acad., 47 (1971), 950-955. 